Proceedings of the 12th International Conference "Ion Implantation and Other Applications of Ions and Electrons", ION 2018

\title{
Modification of MIS Devices by Radio-Frequency Plasma Treatment
}

\author{
D.V. Andreev ${ }^{a}$, G.G. BondAREnKo ${ }^{b}$, V.V. AndreEV ${ }^{a, *}$, \\ V.M. MASLOVSKY ${ }^{c}$ AND A.A. Stolyarov ${ }^{a}$ \\ ${ }^{a}$ Bauman Moscow State Technical University, the Kaluga branch, 2, Bazhenov Str., Kaluga 248000, Russia \\ ${ }^{b}$ National Research University Higher School of Economics, 20, Myasnitskaya Str., Moscow 101000, Russia \\ ${ }^{c}$ Moscow Institute of Physics and Technology (State University), \\ 9, Institutskii per., Dolgoprudnyi, Moscow region 141700, Russia
}

\begin{abstract}
The paper considers an influence of different kinds of radio-frequency plasma treatments onto modification of MIS structures with a thermal $\mathrm{SiO}_{2}$ film which is aimed at improvement of electro-physical parameters of the film. It was found that for the modification of MIS structures it is more preferable to utilize the oxygen plasma radio-frequency plasma treatment performed by a setup with the parallel-plate-type reactor. This is due to the fact that setup allows to have lesser degradation of charge characteristics of the gate dielectric in comparison with a setup with the cylindrical quartz reactor. The radio-frequency plasma treatment stimulates restructuring of $\mathrm{SiO}_{2}$ film and, as a result, diminishes possibility of sample breakdown and raises injection and radiation stability of the samples.
\end{abstract}

DOI: 10.12693/APhysPolA.136.263

PACS/topics: 72.20.Ht, 72.20.Jv, 73.20.At, 73.40.Qv, 73.40.Ty, 77.22.Jp, 77.55.-g

\section{Introduction}

Nowadays the radio-frequency $(\mathrm{RF})$ plasma treatment is broadly utilized to improve characteristics of MOS devices [1-11]. One of its advantages is a possibility to use it in the final stage of manufacturing of integrated circuits after the metallization formation. Papers [2-9] demonstrate that the radio-frequency plasma treatment allows to obtain both thermal annealing of the MOS device and non-thermal processes of neutralization, as well as annealing of charge in the dielectric and at the interface. As a result, at the $\mathrm{RF}$ plasma treatment, a reconstruction of silicon dioxide and $\mathrm{Si} / \mathrm{SiO}_{2}$ interface can take place. A promising tendency of modification of MIS structures by RF plasma treatment is a creation of structures with high stability against influence by high-fields, ionizing radiation, and other stresses. The reconstruction allows to obtain an improvement of MOS device characteristics.

This paper considers change of charge characteristics of MIS structures depending on parameters and modes of RF plasma treatment and their influence on subsequent stability of MIS structures against stress by high-field electron injection into the gate dielectric.

The experiment was implemented for the MOS capacitors which have been a part of test modules located on the silicon CMOS IC wafers. Integrated circuits were manufactured on the $\operatorname{Si}\langle 100\rangle n$-type wafers of the resistivity $4.5 \Omega \mathrm{cm}$. A silicon dioxide film with a thickness of $65 \mathrm{~nm}$ was thermally grown, using dry oxidation at $1000^{\circ} \mathrm{C}$ with $3 \% \mathrm{HCl}$. As the gates, the polysilicon

*corresponding author; e-mail: vladimir_andreev@bmstu.ru
$\left(\mathrm{Si}^{*}\right)$ film, $0.6 \mathrm{~mm}$ thick, doped with phosphorus up to $20 \Omega$ /square, was used. A contact to the substrate and the gate was implemented using the aluminum metallization. The whole surface without contacts of the wafers was coated by a protective film of silicon dioxide of $0.6 \mu \mathrm{m}$ thickness and by a photoresist layer of $1.2 \mu \mathrm{m}$ thickness.

Radio frequency plasma treatment was conducted in a standard commercial plant at a frequency of $13.6 \mathrm{MHz}$ in the modes providing photoresist etching. The photoresist etching in the oxygen RF plasma is utilized as a part of standard CMOS technology and as discovered earlier [11, 12], this can result in silicon device characteristics improvement. For the cylindrical quartz reactor a mixture of $90 \%$ of oxygen and $10 \%$ of nitrogen under $250 \mathrm{~Pa}$ pressure and generator power of $150 \mathrm{~W}$ was used. For the parallel-plate-type reactor oxygen plasma under $10 \mathrm{~Pa}$ pressure and generator power of $400 \mathrm{~W}$ was applied. During treatment of the silicon wafers in the parallel-plate-type reactor the wafers were located on the grounded electrode. The maximum time period of wafer treatment was about 16 min. In the case of the cylindrical quartz reactor the photoresist etching took $8 \mathrm{~min}$. At the same time in the case of the parallel-plate-type reactor the process took 4 min. The charge state of MIS structures change was monitored exploiting high-frequency $C-V$ method and that of multilevel current stress allowing the Fowler-Nordheim high-field tunnel injection of electrons from silicon to take place in the mode maintaining constant current density or step-by-step raising of current stress. At the time of application of current stress, time dependence of voltage across the MIS structure characterizing changes of charge state of MIS structure was measured [11, 13, 14]. 


\section{Experimental results and discussion}

It was found out that RF plasma treatment could result on creation of positive charge in the bulk of the gate dielectric which was a cause of an undesirable shift of threshold voltage of MIS devices. Figure 1 shows the dependences of shift of the band-gap middle voltage $(1,3)$ which were obtained from $C-V$ characteristics and the dependences of voltage shift at the constant injection current $(2,4)$, on time of RF plasma treatment of MIS structures for different reactor types. Figure 1 demonstrates that the RF plasma treatment implemented by the means of the cylindrical quartz reactor results in creation of positive charge in the bulk of $\mathrm{SiO}_{2}$ film which causes the shift of $C-V$ curves (curve 3) to the field of negative voltages and a decrease of $V_{I}$ voltage (curves 2 and 4 ). Thus, that can be neglected by the raising of density of surface states. RF plasma treatment implemented by the means of the parallel-plate-type reactor also causes creation of positive charge in the bulk of $\mathrm{SiO}_{2}$ film (Fig. 1, curves 2 and 4). However, its amount is almost ten times lower than that for the treatment in the cylindrical quartz reactor and, besides, does not result in MIS device characteristics degradation. An equality of curves 1 and 2 is considered as uniform distribution of density of initial dielectric charge by the area. At the same time the presence of the difference (curves 3 and 4 ) with treatment time increase is correlated with nonuniformity of charge surface density resulting distribution in creation of channels with increased density of tunnel current in the dielectric. This fact results in greater changes of $V_{I}$.

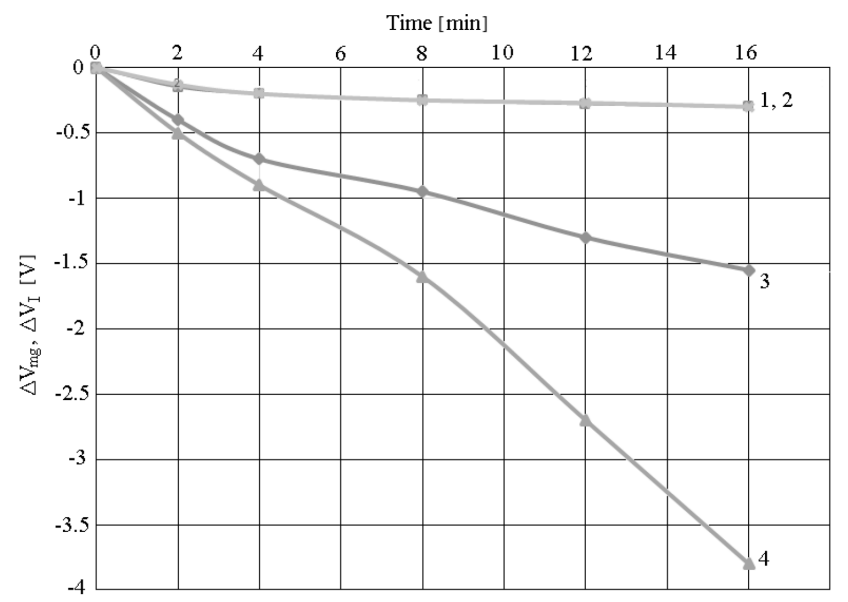

Fig. 1. Dependences of shift of middle gap voltage $(1,3)$ and voltage shift under constant current injection $(2,4)$ on time of the plasma treatment of MIS structure for the parallel-plate-type reactor $(1,2)$ and the cylindrical quartz reactor $(3,4)$.

The RF plasma treatment can have significantly influence on changing charge characteristics of MIS structures at subsequent high-field injection influences. Figure 2 shows the dependences of voltage shift across the MIS structure at high-field injection of electrons by constant current with the density of $10 \mu \mathrm{A} / \mathrm{cm}^{2}$ on the amount of injected charge for the samples treated with different kinds of RF plasma. Figure 2 also shows that for the samples not treated by RF plasma one observes negative shifts of voltage across the sample (curve 0). The fact indicates that an accumulation of positive charge in the dielectric takes place and it is in good agreement with the literature and our previous studies [11, 12, 15-17]. For the MIS structures treated by means of the cylindrical quartz reactor (Fig. 2, curves 3 and 4) one observes electron capture on the traps created by the plasma. For the MIS structures treated by means of the parallel-plate-type reactor after $4 \mathrm{~min}$ of the treatment (Fig. 2, curve 1) a lowering rate of positive charge generation takes place due to creation of electron traps in the $\mathrm{SiO}_{2}$ film. For the MIS structure treated for $8 \mathrm{~min}$ (Fig. 2, curve 2) the density of electron traps created by the plasma rises and the electron capturing on traps becomes the dominant process of charge state change. The fact is demonstrated by positive shift of $\Delta V_{I}$. Accumulating electrons onto the charge traps created by the plasma in the $\mathrm{SiO}_{2}$ film is well described by the following equation [18]:

$$
Q_{\text {trap }}(t)=\sum_{i=1}^{n} Q_{0 i}\left(1-\exp \left(-\frac{\sigma_{i} Q_{\text {inj }}(t)}{q}\right)\right),
$$

where $Q_{0 i}$ is the density of the charge in the dielectric in the case of total occupation of electron traps with the cross-section of $\sigma_{i}, Q_{\text {inj }}$ is the density of injected charge, and $q$ is the electron charge. In our experiment $n$ did not exceed 3 .

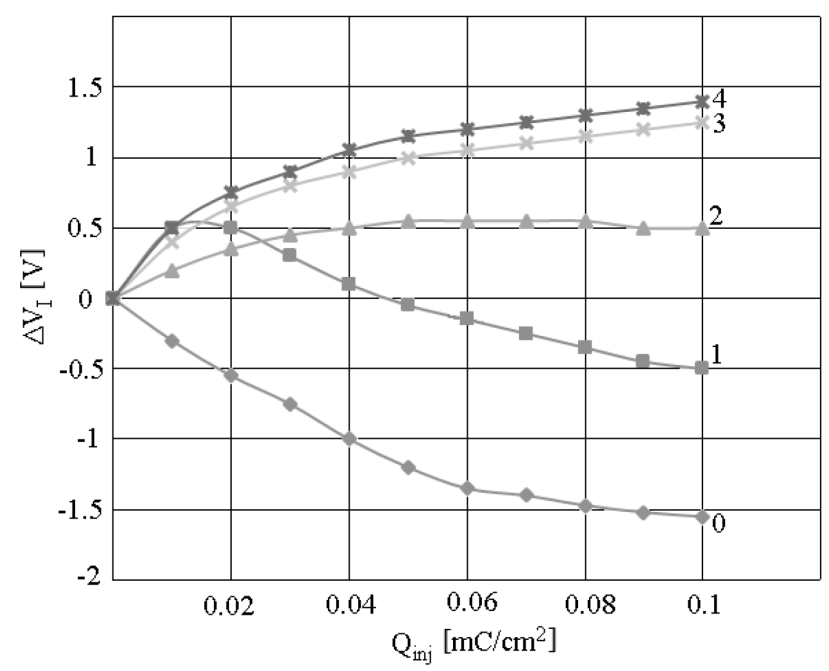

Fig. 2. Dependences of voltage shift on the MOS structure under constant current injection on the amount of charge injected for the following structures: 0 - without plasma treatment; 1,2 - after the RF plasma treatment using the parallel-plate-type reactor; 3, 4 - after the RF plasma treatment using the cylindrical quartz reactor. Time of treatment was as follows: $1-4 \min ; 2,3-8 \min ; 4-16 \min$. 
Calculated from an analysis of experimental data shown in Fig. 2, in accordance to [16-18], the crosssections of electron traps, created by the plasma in the $\mathrm{SiO}_{2}$ film, are in the range of $2 \times 10^{-15}-10^{-16} \mathrm{~cm}^{2}$. Reference [12] demonstrates that the arc plasma jet treatment creates electron traps with the cross-sections ten times lower than that for the traps correlated with the hydroxyl groups.

Thus it was found out that for modification of MIS structures it was more preferable to utilize RF plasma treatment realized by means of the parallel-plate-type reactor because it allowed to have lower degradation of the gate dielectric (voltage shift of the middle of bandgap is $0.1-0.3 \mathrm{~V}$ ) in comparison with the cylindrical quartz reactor (voltage shift of the middle of bandgap is $1-3.5 \mathrm{~V})$.

Figure 3 shows the histograms of MIS structure distribution by the charge injected until the dielectric breakdown $\left(Q_{\mathrm{bd}}\right)$ for the samples not treated by the plasma (1) and after the RF plasma treatment by means of the parallel-plate-type reactor for $6 \mathrm{~min}(2)$.

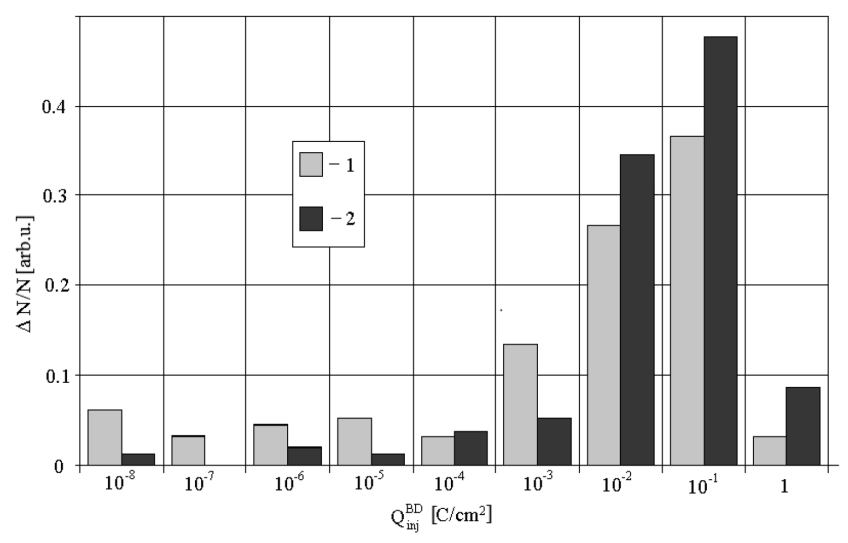

Fig. 3. Histograms of the MOS structures distribution by the charge-to-breakdown for the samples before plasma treatment (1) and after plasma treatment (2) by means of the parallel-plate-type reactor for $6 \mathrm{~min}$.

Figure 3 demonstrates that the treatment of MIS structures by oxygen plasma by means of the parallel-platetype reactor for 5-10 min allows to create in the bulk of $\mathrm{SiO}_{2}$ film a density of electron traps sufficient to compensate the effect of positive charge generation during high-field injection. As a result, in the initial stage of tunnel Fowler-Nordheim injection of electrons from the silicon in the high-fields, the trapping of electrons in the $\mathrm{SiO}_{2}$ film becomes the dominating process of charge state change and differs for generation of positive charge for the non-treated structures. The fact reduces a possibility of samples breakdown and increases their injection and radiation stability.

In the case of influence by the RF plasma onto the MIS structures, low-energy ions of oxygen and electrons most frequently do not have sufficient energy to penetrate through the gate and thus the photons with the energy higher than $1 \mathrm{keV}$ most significantly influence the gate dielectric. Simultaneous influence of UV, X-ray, and besides, alternate RF field onto the MIS structures cause processes of ionization in the gate dielectric and injection of electrons and holes from the electrodes into it. Due to these effects one can observe processes of $\mathrm{Si}-$ $\mathrm{H}$ and $\mathrm{Si}-\mathrm{OH}$ bond breakage in the $\mathrm{SiO}_{2}$ film which results in creation of electron traps in the dielectric bulk $[2,4,7]$. Electron traps with similar cross-sections were also observed in the Si film when the MIS structures were treated with hydrogen RF plasma [2]. Hence $\mathrm{RF}$ plasma influence is in many respects identical to the radiation-thermal treatment [19-21] and by causing processes of hydrogen redistribution in the $\mathrm{SiO}_{2}$ film, it can result in reconstruction of structure of the dielectric film, lowering defectiveness, and eliminating strained chemical bonds. A verification of this assumption are the experimental data shown in Fig. 3. They demonstrate that the RF plasma treatment allows to reduce a number of defective structures with low charge stability.

\section{Conclusions}

It was found out that in order to modify the MIS structures it is more preferable to utilize oxygen RF plasma treatment realized by means of the parallel-platetype reactor since it allows to obtain lower degradation of gate dielectric in comparison with the treatment performed using the cylindrical quartz reactor. As demonstrated under some conditions of RF plasma treatment by means of the parallel-plate-type reactor due to the interactions of the plasma with hydrogen complex it is possible to obtain a required density of electron traps with the cross-sections of $2 \times 10^{-15}-10^{-16} \mathrm{~cm}^{2}$ in the bulk of $\mathrm{SiO}_{2}$ film. As a result, electron trapping in the $\mathrm{SiO}_{2}$ film becomes the dominating process of the charge state change with high-field tunnel injection of electrons from the silicon. The effect results in reducing of possibility of sample breakdown. This differs from the case of non-treated structures for which generation of positive charge is the dominating process. Besides, the RF plasma treatment stimulates reconstruction of the $\mathrm{SiO}_{2}$ film which results in lowering its defectiveness and eliminating strained chemical bonds. This causes rise of injection and radiation stability of the MIS devices.

\section{Acknowledgments}

This paper was supported by the Ministry of Education and Science of the Russian Federation by the state task of Bauman Moscow State Technical University (Project No. 8.6779.2017/8.9). Support from the Basic Research Program of the National Research University Higher School of Economics is gratefully acknowledged. 


\section{References}

[1] T.P. Ma, W.H.L. Ma, Solid State Electron. 22, 663 (1979).

[2] A.N. Nazarov, V.S. Lysenko, T.M. Nazarova, Semicond. Phys. Quant. Electron. Optoelectron. 11, 101 (2008).

[3] A.N. Nazarov, V.I. Kilchytska, I.P. Barchuk, A.S. Tkachenko, S. Ashok, J. Vac. Sci. Technol. B 18, 1254 (2000).

[4] J. Arreola, M. Keusgen, M.J. Schoning, Phys. Status Solidi A 214, 1700025 (2017).

[5] G.T. Pan, S. Chong, T.C. Yang, Y.L. Yang, N. Arjun, Chem. Eng. Commun. 203, 1666 (2016).

[6] K. Nagata, H. Akamatsu, D. Kosemura, T. Yoshida, M. Takei, M. Hattori, A. Ogura, T. Koganezawa, M. Machida, J. Son, I. Hirosawa, T. Shiozawa, D. Katayama, Y. Sato, Y. Hirota, ECS Trans. 19, 45 (2009).

[7] A. Szekeres, S. Alexandrova, Phys. Status Solidi A 77, 721 (1983).

[8] V.K. Sunny, V.N. Mishra, R. Dwivedi, R.R. Das, IEEE Sens. J. 16, 6205 (2016).

[9] J. Gong, J. Xu, L. Liu, H. Lu, X. Liu, Y. Feng, J. Semicond. 38, 094004 (2017).

[10] L.S. Wang, J.P. Xu, L. Liu, IEEE Trans. Electron. Dev. 62, 1235 (2015)

[11] V.V. Andreev, G.G. Bondarenko, V.M. Maslovsky, A.A. Stolyarov, Acta Phys. Pol. A 125, 1371 (2014).
[12] V.V. Andreev, G.G. Bondarenko, V.M. Maslovsky, A.A. Stolyarov, Acta Phys. Pol. A 128, 887 (2015).

[13] V.V. Andreev, G.G. Bondarenko, V.M. Maslovsky, A.A. Stolyarov, D.V. Andreev, Phys. Status Solidi C 12, 299 (2015).

[14] V.V. Andreev, G.G. Bondarenko, V.M. Maslovsky, A.A. Stolyarov, D.V. Andreev, Phys. Status Solidi C 12, 126 (2015).

[15] D. Arnold, E. Cartier, D.J. DiMaria, Phys. Rev. B 49, 10278 (1994).

[16] A. Strong, E. Wu, R. Vollertsen, J. Sune, G. Rosa, S. Rauch, T. Sullivan, in: Reliability Wearout Mechanisms in Advanced CMOS Technologies, IEEE Press Series on Microelectronic Systems, Wiley, Hoboken (NJ) 2009

[17] D.M. Fleetwood, Microelectron. Reliabil. 80, 266 (2018).

[18] D.V. Andreev, G.G. Bondarenko, V.V. Andreev, V.M. Maslovsky, A.A. Stolyarov, High Temp. Mater. Process. Int. Quart. High-Technol. Plasma Process. 21, 299 (2017).

[19] D.M. Fleetwood, IEEE Trans. Nucl. Sci. 65, 1465 (2018).

[20] G.M. Voronkova, V.D. Popov, G.A. Protopopov, Semiconductors 41, 958 (2007).

[21] D.V. Andreev, G.G. Bondarenko, V.V. Andreev, V.M. Maslovsky, A.A. Stolyarov, Acta Phys. Pol. A 132, 245 (2017) 